\title{
Lessons Learned from Running a Conference in the Time of COVID-19 and the Silver Linings of Shifting to Online
}

\author{
By Hannah E. Power, Madelaine S.S. Broadfoot, Annette Burke, Paul M. Donaldson, Rosanne M. Hart, \\ Kendall C. Mollison, David J. Schmidt, and Stuart M. Young
}

\section{INTRODUCTION}

Conferences play a critical role in the scientific process as places where researchers can disseminate results of their studies and make new contacts or strengthen those already formed. They are particularly vital for students and early career researchers and practitioners because they provide opportunities to showcase skills to established members of the community and allow for networking, two activities that are often vital for securing future employment and career support. The COVID-19 pandemic resulted in the cancellation or postponement of many conferences, reducing these crucial, career-enhancing experiences for the next generation of scientists and engineers.

The Young Coastal Scientists and Engineers Conference (YCSEC) was initiated in the UK in 2005 to bring together early career scientists and engineers who investigate the physical and biological processes within the coastal environment. It has been successfully run in the UK annually since inception, and the concept has been adopted via the YCSECAmericas, held annually since 2014. In 2019, a similar conference series was proposed for Australia, with the goal of holding the inaugural Australian YCSEC (AusYCSEC) in Newcastle in June 2020. The mission statement developed for the conference series was "Connecting, fostering, and showcasing the next generation of Australian coastal scientists and engineers." A local organizing com- mittee (LOC) of students and early- to mid-career researchers was established to ensure this mission was met. The LOC was run on a volunteer basis to keep costs low and provide an affordable and accessible conference for the Australian coastal community. In March 2020, as COVID-19 spread around the world, it became clear that holding an in-person conference in June would not be feasible. Hence, the conference was transitioned to online.

Here, we describe how this volunteerrun conference was rapidly reimagined to an online format while meeting, and in some aspects exceeding, its original aims and mission. We detail the considerations required for transitioning the conference from in-person to online, the session types and how they were adapted to maximize impact in an online setting, and the outcomes and positive lessons learned from the process.

\section{CHALLENGES OF COING ONLINE}

The inaugural AusYCSEC event was planned as an in-person, three-day conference to be held at the University of Newcastle (UON), NSW, Australia, with an anticipated attendance of 60-80 people. With large in-person gatherings not allowed due to COVID-19, the conference was transitioned to online.

Several challenges had to be overcome in converting the standard in-person conference program to an online format. Foremost among them was keeping the event engaging for online dele- gates. Recognizing from recent remote working and teaching experiences that online concentration spans could only be maintained for 90-minute to twohour blocks, the LOC decided it would not be desirable to simply transfer the original all-day, in-person schedule to an online format. Cognitive loads associated with online interaction are much greater than those of an in-person conference setting. Thus, an early decision was made to adopt shorter sessions, with most sessions limited to one hour and no sessions exceeding two hours. All sessions were separated by extended breaks to allow delegates to attend to other obligations and avoid lengthy online sessions. Further, the program was designed to encourage those working remotely to attend most (if not all) conference sessions without losing entire days of their work or research schedules. The resulting limited session times presented other challenges, as they required strategic and selective decisions regarding which sessions to retain and how they could be adapted. Sessions were scheduled at times identified as most suitable for potential attendees via an online survey tool.

The decision to shift to online required the selection of an online video conferencing platform. While the LOC were software agnostic, Zoom was ultimately selected as it was fast becoming one of the most widely used platforms for online meetings and was accessible via an institutional license provided by UON. 
Further, its functionality was desirablewith both Zoom Webinar and Zoom Meeting modes providing features that supported the different styles of conference sessions planned. The LOC held several practice Zoom sessions to explore the platform, troubleshoot issues, and identify hosting and technical roles. These pre-planning and practice sessions were crucial for ensuring that presentation, interaction, and feedback functions were seamless for presenters and attendees. Presenters were also requested to attend practice sessions to receive guidance on the structure and logistical formatting of their formal sessions.

The LOC ran the three-day online conference from a UON teaching space of sufficient size to accommodate physical distancing for those present. This allowed for concise and effective communication both before and during live sessions, resulting in a seamless and professional experience for conference attendees. A rotating roster of Zoom roles (including backups) further facilitated smooth sessions by allowing technical glitches to be promptly resolved without affecting the attendee experience.

Social media platforms were used to enhance the reach of the new conference and to promote key information, such as significant dates, keynote speakers, and website URLs. An electronic conference program with embedded session-specific Zoom links was created to facilitate fast and easy attendee access to each session. Zoom links and calendar invites were also provided in the days leading up to the conference, further facilitating a prompt and simplified process for joining each session.

\section{CONFERENCE SESSION TYPES AND FORMATS}

Moving online meant rethinking conference session formats. The goal was to provide a short, punchy, and engaging online program that did not sacrifice conference content, despite the constraints of online delivery. The adopted program, which featured reimagined versions of the planned in-person sessions, consisted of four technical session formats: the opening keynote address, express courses, lightning presentations, and a closing panel discussion. There was also one social, speed-networking session.

We used the Zoom Webinar feature for the technical sessions. It provided video participation for session chairs and presenters/panelists, with delegates limited to a "view, listen, and message" mode. The technical sessions retained many standard conference elements. Session chairs provided introductions, managed time, and asked/facilitated questions; presenters/panelists presented their slides and work and/or contributed to panel discussions.

The networking session used Zoom Meeting, which allowed all participants to communicate and interact via video, audio, and messaging.

\section{Keynote Address}

The conference keynote was the first session of the online conference. Given that only one keynote session was planned, the speaker was chosen to cover a topic that most early career coastal scientists and engineers would not have had much exposure to but that would be highly relevant to all: the legal and financial liability associated with climate change and how it can be a driver to effect change. The keynote address was presented by an industry professional, giving students some insight into careers in industry.

\section{Lightning Presentations}

The condensed online program meant that the time available for presentations was significantly reduced. However, the LOC was committed to providing a platform for all who had submitted abstracts for the in-person conference, in addition to facilitating additional abstract submissions for those who could now attend the online conference. The standard inperson, 15-minute presentation format was no longer feasible, so the "ThreeMinute Thesis" (elevator pitch) model was chosen to maximize the number of presentations per session, while still using a delivery convention familiar to most.

Four 60-minute "lightning presentation" sessions were scheduled, each covering a different theme. Presenters were allocated five minutes: three minutes to present their work (one slide only) and two minutes for questions. A PowerPoint slide template was provided to presenters, with the final presentation returned to the LOC prior to the conference. The LOC compiled slide sets for each session to ensure smooth transitions between presenters and avoid time delays or technical issues that could have arisen had presenters been required to share their screens.

A timer feature was placed on each slide to help manage the tight, non-standard timeframes. This allowed both the presenter and LOC session chair to monitor the time and ensured sessions were kept to schedule. Sessions were run in a similar way to those in an in-person conference format, with a session chair introducing the session and theme and each presenter in turn. Attendees were informed of the format of the session, the general rules, and the process for asking questions at the beginning of each session.

The lightning presentation format resulted in brief and engaging presentations that showcased key aspects of presenters' research in an informal environment and reached a broader audience than would have been possible at a traditional in-person conference. Attendees used the chat function to provide feedback and ask questions. The LOC was able to communicate directly with attendees and respond to questions within the chat.

\section{Express Courses}

Several pre-conference workshops were originally planned to provide delegates with in-depth learning opportunities. Recognizing that all-day workshops were not suited to an online format, the subject matter and learning content were transitioned using the format of express courses-short seminar/ introductory "how to" types of professional courses. The intent was to provide 
attendees with a practical introduction and learning experience on a topic that would enable them to undertake further independent learning should they wish to. Again, online engagement and interaction was high, with delegates using the chat and Q\&A messaging tools.

\section{Panel Discussion}

The online conference closed with a panel discussion on "how to navigate a career in coastal geoscience and engineering," designed to benefit the core target audience. The session provided information to early career researchers/professionals on the wide variety of potential career pathways available within coastal geoscience and engineering and enabled panelists to share their advice on navigating a career in these varied fields based on their experiences. The four panelists were selected to represent a broad cross section of the coastal geoscience and engineering: a variety of fields (science, engineering, and management) and sectors (academic, government, consulting, and small business), male and female (two women, two men), and different career stages (middle to established). The session proved to be highly interactive, with the four panelists answering live questions from the chair, who relayed delegate questions posted via the Q\&A tool.

\section{Interactions and Engagement}

The opening keynote, express course, lightning presentation, and panel discussion sessions were all held using Zoom Webinar. One primary benefit of Zoom Webinar is that it permits differentiation between hosts/panelists and attendees, with the latter unable to turn on their webcams or microphones, thus preventing interruptions. It also has a built-in Q\&A tool that allows attendees to pose questions for direction to the speaker by the session chair. The ability to up-vote questions provides session chairs with the ability to prioritize the most popular questions, allowing the presenters to focus on their responses.

Attendees could also use the text- based chat function for discussion or informal communication without interrupting the flow of the session. The use of the chat tool was encouraged by the session chairs and promoted by LOC members, who posted comments and openended questions that sought participant feedback and helped foster engagement. While Zoom Webinar would likely scale well for larger events, disadvantages include a lack of transparency for attendees regarding who else is attending the session and the number of attendees.

\section{Speed Networking}

Conference networking was the in-person conference aspect that needed the most inventive solution in the transition to online. For early career researchers and students, networking is an invaluable aspect of attending conferences and was a key goal of this conference. The solution was formed from the idea of marrying speed dating and online "breakout room" concepts that committee members had used in recent teaching experiences. The 60-minute networking session was run through Zoom Meeting, as opposed to Zoom Webinar, and consisted of four breakout room rotations. Attendees commenced the session in the "main room" where details of the session were explained. Attendees were then randomly assigned to breakout rooms with four to five others for approximately 15 minutes. This amount of time was sufficient to enable all attendees in a room to introduce themselves and have a brief discussion to enable networking, while also keeping the session dynamic. The session ran toward the end of the conference schedule so that many attendees had already given lightning presentations and were known to others. Between each breakout room rotation, all attendees were brought back together in the main room, enabling the LOC to maintain communications with everyone. The LOC also had the ability to move between rooms and, therefore, to moderate all of them. All attendees eventually returned to the main room for the conclusion of the session.

\section{CONFERENCE OUTCOMES}

Attendance at the inaugural AusYCSEC event exceeded expectations; more than double the number of participants originally estimated for an in-person conference joined the three-day online event for a total of 178 unique attendees. These numbers compare well to other, more mature coastal conference series in Australia (e.g., the 28th Annual NSW Coastal Conference attracted 250 delegates in 2019). Further, attendance numbers did not decline as the conference progressed, with an average of 72 (st. dev. =15) attendees in each session.

The online platform increased accessibility by removing or decreasing barriers typically associated with in-person conferences, including geographical distances, time commitments, and financial costs. The decision to remove registration fees was made to increase attendance/ engagement in the conference series. This was possible due to the minimal costs associated with running an online event (e.g., no catering expense). Additionally, as the conference was the inaugural event, holding the first iteration freely online provided publicity and a platform for increasing the success of future, in-person AusYCSEC conferences.

Original expectations were that the inperson conference would attract mostly local to regional delegates; however, transitioning online significantly extended its geographical reach with attendees joining from Oceania, Asia, Europe, North America, and South America. The majority of the attendees were Australian based, with good representation from New Zealand (6\%) and Indonesia (3\%). For other conferences being held online that are aiming for a more global reach, further consideration would be required for the scheduling of any synchronous sessions.

Overall, the conference was positively received by attendees, with $100 \%$ of survey respondents rating the conference as either "good," "great," or "excellent," and $100 \%$ indicating they would attend future conferences in this series $(n=35)$. 
Feedback reflecting the overall positive response included:

- "Short talks were awesome, ran really smoothly and not death by Zoom!”

- "The panel discussion was excellent, and I appreciated the seamless coordination of an online event. This was one of the most professionally run online events I have attended since we began working remotely. Well done!"

- "So well organized. Great topics including breadth. The format really worked, including the speed networking, and the way the IT was handled was very professional. Best I have seen in this new world."

Constructive feedback was also received, with some finding the lighting presentations too compressed and others commenting that they would have preferred to know who else was in attendance during the Zoom Webinar-based sessions. Despite this feedback, the collective response was generally supportive of the adopted format.

A key, long-term goal of the AusYCSEC series is to provide a new and sustainable forum that specifically benefits the next generation of coastal scientists and engineers in Australia. The success of the inaugural event has set the conference series in good stead toward achieving this goal.

\section{LESSONS LEARNED AND THE BENEFITS OF COING ONLINE}

While metrics and feedback are important, they don't necessarily provide an evaluation of performance against strategy or goals. With the overarching mission of the AusYCSEC conference series to "connect, foster, and showcase the next generation," it's imperative to ask if these aims were achieved and if the online format helped or hindered attaining them.

\section{Connecting the Next Generation}

Networking, a key benefit of conferences typically happens during social events, session breaks, and on the sidelines of in-person conferences. An online conference does not provide these physical settings to facilitate networking, so a key challenge with transitioning online was to provide virtual networking opportunities. The "speed networking" session was designed to fill this gap and was well attended and well received. Many attendees noted how the structured session, with randomly allocated small breakout groups and loosely structured talking points, provided an easy framework for meeting others regardless of their career levels or areas of expertise. Advantageously, it also removed some of the challenges associated with engaging with other, unfamiliar attendees in an unstructured social setting such as those common at in-person conferences with attendees indicating that they met people they would not have felt comfortable introducing themselves to at an in-person event. The random allocation to breakout rooms encouraged networking with people in different fields, further removing barriers within the community. This format would likely work well for any group wanting to foster connections between members.

\section{Fostering the Next Generation}

Providing a supportive environment that develops skills and expertise, disseminates knowledge, and provides mentoring opportunities is critical for a conference that targets students and early career researchers and practitioners. The conference content and program were curated to provide opportunities for attendees to build skills through the express courses, gain knowledge in cutting-edge research through the lightning presentation sessions, and receive career guidance through the panel discussion. In particular, the panel discussion provided attendees with advice on a range of topics, including career progression, application of coastal knowledge to technical and policy challenges, essential "soft" and "hard" skillsets, and the importance of networks/relationships. Both the panel discussion and the technical workshops benefited from the online format, allowing the LOC to look further afield for pre- senters and panelists who would otherwise have been unable to present at an in-person conference, resulting in more varied and internationally significant sessions for the attendees.

\section{Showcasing the Next Generation}

Showcasing the work of the next generation of coastal scientists and engineers is a key goal of this conference series. The transition to online greatly increased attendance numbers and the reach of the conference in terms of the geographical locations and career progressions of attendees, thus enhancing the exposure of students, early career researchers, and young professional presenters and their work. Unexpectedly, the conference received strong engagement from senior members of the Australian coastal community, with several indicating they would have been unlikely to attend an in-person event due to time and financial commitments but happily attended the online event due to easier access. This further enhanced the exposure of the presenters, as conferences of this type (i.e., those primarily targeting students and early career researchers and practitioners, which often focus on networking) are typically less appealing to senior members of the community. In summary, the online format increased the size and breadth of the audience, thus helping to further showcase work from the next generation.

\section{Other Factors of Success}

Several other factors also likely contributed to the success of the online conference, including: (1) attendees' familiarity with online video conferencing platforms after several months of remote working, (2) existence of an engaged and enthusiastic target audience, (3) a clear gap in the conference market because of the pandemic, (4) a local desire for an event specifically targeted at students and early career researchers and practitioners, and (5) a committed volunteer LOC who contributed time, effort, and energy to ensure goals, targets, and deadlines were met. 


\section{WHAT NEXT?}

The challenging process of transitioning the conference online would not have occurred without necessity. However, the significant advantages and positive lessons learned from this online format will be taken forward as this conference series evolves. The online format clearly has benefits such as those described above, along with others such as reduced carbon footprints (e.g., Klöwer et al., 2020). The novel format was appreciated by the vast majority of attendees, with $97 \%$ of those who provided feedback indicating they would like to see future conferences have either a mixed online/in-person format or have the conference series toggle between formats in alternating years. However, there was also clear feedback from attendees that future in-person events in this conference series would be popular (100\% of survey respondents said they would attend an in-person event next year if one could be held). Given this feedback, future conferences in this series will likely always incorporate some online component. One example of a mixed online/in-person format could be a series of short sessions held in person but streamed online to enable wider engagement and further showcase students and early career researchers as the schedule and sessions described here did. For in-person attendees, the longer gaps could be filled with opportunities for extended presentations (e.g., over accompanying posters), field trips, and structured networking (e.g., an in-person replication of the speed networking described here). Whether we can hold an in-person conference in 2021 remains to be seen, but the lessons learned from running the first AusYCSEC online have certainly changed how this conference series will be run in future, resulting in conferences that are more accessible and that better meet their goals and objectives. 응

\section{REFERENCE}

Klöwer, M., D. Hopkins, M. Allen, and J. Higham. 2020. An analysis of ways to decarbonize conference travel after COVID-19. Nature 583:356-359, https://doi.org/10.1038/d41586-020-02057-2.

\section{ACKNOWLEDCMENTS}

The authors acknowledge those who initially conceived the YCSEC-UK Series (Alistair Borthwick, Jenny Brown, Nicholas Dodd, Suzana Ilic, Robert Nicholls, and Richard Simons) and particularly thank Chris Blenkinsopp, Ryan Mulligan, and Jack Puleo fo advice in bringing this conference series to Australia. The authors thank the Australian Coastal Research Network Steering Committee of Andrew Pomeroy, Mike Kinsela, and Tom Murray for their support and also the many students and early career researchers and practitioners who helped promote the conference. We thank the University of Newcastle for the online hosting of the conference. Sponsorship for the conference was provided by the Office of the NSW Chief Scientist \& Engineer, within the NSW Department of Planning, Industry \& Environment under the Conference Sponsorship Program 2020 Lastly, we thank those who contributed their time and energy to the conference sessions, those who presented their work, and all those who attended. In particular, we thank the keynote speaker, Natalie Bentley; the express course presenters, Brittany Bruder and Gareth Davies; and the panel members, Matt Eliot, Tom FitzGerald, Michelle Fletcher, and Kristen Splinter.

\section{AUTHORS}

Hannah E. Power (hannah.power@newcastle.edu.au) is Senior Lecturer, School of Environmental and Life Sciences, University of Newcastle, Callaghan, New South Wales (NSW), Australia. Madelaine S.S. Broadfoot is Environmental Scientist, BMT, Newcastle, NSW, Australia. Annette Burke is PhD Candidate, School of Earth and Environmental Sciences, Macquarie University, Ryde, NSW, Australia. Paul M. Donaldson is Senior Estuary Management Officer, NSW Central Coast Council, Wyong, NSW, Australia. Rosanne M. Hart is PhD Candidate, Kendall C. Mollison is PhD Candidate, and David J. Schmidt is Honours Student, all in the School of Environmental and Life Sciences, University of Newcastle, Callaghan, NSW, Australia. Stuart M. Young is Senior Coast and Estuaries Officer, NSW Department of Planning, Industry and Environment, Newcastle, NSW, Australia.

\section{ARTICLE CITATION}

Power, H.E., M.S.S. Broadfoot, A. Burke, P.M. Donaldson, R.M. Hart, K.C. Mollison, D.J. Schmidt, and S.M. Young. 2020. Lessons learned from running a conference in the time of COVID-19 and the silver linings of shifting to online. Oceanography 33(3):21-25, https://doi.org/10.5670/ oceanog.2020.312.

\section{COPYRICHT \& USACE}

This is an open access article made available under the terms of the Creative Commons Attribution 4.0 International License (https://creativecommons.org/ licenses/by/4.0/), which permits use, sharing, adaptation, distribution, and reproduction in any medium or format as long as users cite the materials appropriately, provide a link to the Creative Commons license, and indicate the changes that were made to the original content.

\author{
A Call for \\ Short Articles
}

Share a successful outcome

of a "broader impacts" activity

as well as any lessons learned

during the project's development

and execution. We are

interested in compiling stories

that may serve as templates

for other researchers who are

embarking on education and

public outreach projects.

Follow the instructions for

Commentaries, Meeting Reports,

and Ocean Education and Ocean

Policy articles at:

\title{
https://tos.org/
}

-ceanography/guidelines

Questions?

Contact Ellen Kappel at

ekappel@geo-prose.com 